\title{
Signal Analysis on the Ball: Design of Optimal Basis Functions with Maximal Multiplicative Concentration in Spatial and Spectral Domains
}

\author{
Wajeeha Nafees*, Zubair Khalid*, Rodney A. Kennedy ${ }^{\dagger}$ \\ ${ }^{*}$ School of Science and Engineering, Lahore University of Management Sciences, Lahore, Pakistan \\ ${ }^{\dagger}$ Research School of Engineering, The Australian National University, Canberra, ACT 2601, Australia \\ 14060020@lums.edu.pk, zubair.khalid@lums.edu.pk, rodney.kennedy@anu.edu.au
}

\begin{abstract}
In this work, we design a set of complete orthonormal optimal basis functions for signals defined on the ball. We design the basis functions by maximizing the product of their energy concentration in some spatial region and that in some spectral region. The optimal basis functions are designed as a linear combination of space-limited functions with maximal concentration in the spectral region and band-limited functions with maximal concentration in the spatial region. The proposed optimal basis functions are shown to form a complete set for signal representation in a subspace formed by the vector sum of the subspaces spanned by space-limited and band-limited functions. We also formulate an integral operator which projects the signal to the joint subspace and maximizes the product of energy concentrations in harmonic as well as spatial domains. Furthermore, with the help of some properties of proposed optimal basis functions we show that these functions are the only eigenfunction of the integral operator.

Index Terms - spatial-spectral concentration, localized spectral analysis, Slepian concentration problem, ball, optimal basis, energy concentration
\end{abstract}

\section{INTRODUCTION}

Spherical signal processing has received tremendous amount of attention over the past two decades as a mainstream tool to explain various phenomena in the fields of geophysics, cosmology, medical imaging and wireless communications (e.g. [1]-[5]). Signals or data are naturally defined on a three-dimensional spherical manifold (solid sphere or ball) in these applications. Recently, several signal processing methods have been developed and extended to support signal analysis in these applications [1], [6], [7]. In this context, we consider a problem to design optimal basis, with optimal (to be defined shortly) concentration in spatial and spectral domains, for the representation of signals defined on the unit ball.

A widely known feature of a signal is that it cannot have finite support in both the spectral (harmonic) and spatial domains at the same time as reported in [8], where the concentration problem (finding band-limited functions or space-limited functions having maximal energy concentration in spatial or harmonic domain, respectively) was solved nearly fifty years ago for the one-dimensional case (time and frequency). Although the concentration problem was first defined in the Euclidean domain, the generalizations of the concentration problem for various geometries, for example sphere [9], ball [10] and two-dimensional space [2], have also been explored. Solution of the concentration problem provides orthogonal family of functions that are maximally concentrated in the spectral (spatial) domain while being exactly limited in the spatial (spectral) domain and have been used extensively for spatial windowing, spectral estimation and localized spectral analysis [11]-[13]. In practice, we encounter band-limited functions that are only accessible over a specific region in space due to physical restrictions, or spacelimited functions that may be band-limited due to practical limitations of the measuring equipment. To support these applications, we consider the problem of maximizing the product of concentration of energy of signals defined on the ball. The problem of finding optimal basis with maximum energy concentration in spatial and harmonic domains, has been considered for Euclidean [14] and spherical (unit sphere) [15] domains.

In this work, we design a set of functions which are maximally and simultaneously concentrated in both the spectral and spatial domains. We call them the optimal basis functions. We consider the vector sum of subspaces spanned by space-limited spectrally concentrated and band-limited spatially concentrated functions which arise as a solution of Slepian concentration problem on the ball [10], and design basis functions for this subspace. Using a weighted linear combination of the band-limited and space-limited eigenfunctions (obtained as a solution of Slepian concentration problem), we design optimal basis functions which maximize the product of concentration of energy in the spectral and spatial domain. We further show that the proposed basis functions serve as complete bases for the representation of a signal in the joint subspace-vector sum of subspaces. We also point out that the proposed basis functions are the eigenfunctions of an integral operator that maximizes the product of energy concentration of the signal in the spectral and spatial domains.

We organize the remainder of the paper as follows. We first look into the mathematical preliminaries necessary to understand this work and the Slepian concentration problem on the unit ball in Section II, where we also formulate the problem under consideration. In Section III, we then present the design of optimal basis functions, formulation of integral operator and analysis of proposed optimal basis functions. Finally, the concluding marks are then made in Section IV. 


\section{Mathematical Preliminaries And Problem FORMULATION}

\section{A. Signals on the Ball}

We consider square-integrable complex-valued functions defined on the ball $\mathbb{B}^{3} \triangleq \mathbb{R}^{+} \times \mathbb{S}^{2}$, where $\mathbb{R}^{+}=[0, \infty)$ on the real line and $\mathbb{S}^{2}$ represents the 2 -sphere. These functions defined on the ball form a Hilbert space $L^{2}\left(\mathbb{B}^{3}\right)$ with an inner product defined as

$$
\left\langle f_{1}, f_{2}\right\rangle \triangleq \int_{\mathbb{B}^{3}} f_{1}(\boldsymbol{r}) \overline{f_{2}(\boldsymbol{r})} d v(\boldsymbol{r}), \quad f_{1}, f_{2} \in L^{2}\left(\mathbb{B}^{3}\right) .
$$

Here $\boldsymbol{r} \equiv \boldsymbol{r}(r, \theta, \phi) \triangleq(r \sin \theta \cos \phi, r \sin \theta \sin \phi, r \cos \theta)^{T}$ $\in \mathbb{R}^{3}$ represents a vector which parameterizes a point on the ball with $r \in \mathbb{R}^{+}, \theta \in[0, \pi]$ and $\phi \in[0,2 \pi)$ denoting radial distance, co-latitude and longitude, respectively. The integration in (1) is carried out over the entire $\mathbb{B}^{3}$ i.e. $\int_{\mathbb{B}^{3}} \equiv \int_{r=0}^{\infty} \int_{\theta=0}^{\pi} \int_{\phi=0}^{2 \pi}, d v(\boldsymbol{r})=r^{2} \sin \theta d r d \theta d \phi$ is the differential volume element on $\mathbb{B}^{3}$ and $\overline{(\cdot)}$ denotes the complex conjugate operation. The inner product given in (1) induces a norm $\|f\| \triangleq\left\langle f_{1}, f_{2}\right\rangle^{1 / 2}$. The functions with finite induced norm (finite energy) are referred to as "signals on the ball". Also, for any region $R \subset \mathbb{B}^{3}$, we define $\left\langle f_{1}, f_{2}\right\rangle_{R} \triangleq$ $\int_{R} f_{1}(\boldsymbol{r}) \overline{f_{2}(\boldsymbol{r})} d v(\boldsymbol{r})$. We also define a linear integral operator $\mathcal{S}$ with kernel $S\left(\boldsymbol{r}, \boldsymbol{r}^{\prime}\right)$ using Fredholm integral equation, that is

$$
\left(\mathcal{S} f_{1}\right)(\boldsymbol{r})=\int_{\mathbb{B}^{3}} S\left(\boldsymbol{r}, \boldsymbol{r}^{\prime}\right) f_{1}\left(\boldsymbol{r}^{\prime}\right) d v\left(\boldsymbol{r}^{\prime}\right) .
$$

\section{B. Fourier-Laguerre Transform and Spectral Representation}

The Fourier-Laguerre basis functions, denoted by $Z_{\ell m p}$, for angular degree $\ell \geq 0$, order ${ }^{1}|m| \leq \ell$ and radial integer degree $p \geq 0$ are chosen for harmonic representation of signals on the ball [1], [10]. These basis functions are defined as

$$
Z_{\ell m p}(\boldsymbol{r}) \triangleq K_{p}(r) Y_{\ell m}(\theta, \phi), \quad \boldsymbol{r} \equiv \boldsymbol{r}(r, \theta, \phi),
$$

where $Y_{\ell m}(\theta, \phi)$ is the spherical harmonic function of degree $\ell$ and order $m$ and $K_{p}(r)$ is defined for non-negative integer radial degree $p$ as

$$
K_{p}(r) \triangleq \sqrt{\frac{p !}{(p+2) !}} e^{-r / 2} L_{p}^{(2)}(r)
$$

with $L_{p}{ }^{(2)}$ representing the $p$-th generalized Laguerre polynomial of second order, defined as

$$
L_{p}{ }^{(2)}(r) \triangleq \sum_{j=0}^{p}\left(\begin{array}{l}
p+2 \\
p-j
\end{array}\right) \frac{(-r)^{j}}{j !} .
$$

Due to completeness of Fourier-Laguerre basis functions [1], we can expand any signal $f \in L^{2}\left(\mathbb{B}^{3}\right)$ as

$$
f(\boldsymbol{r})=\sum_{\ell=0}^{\infty} \sum_{m=-\ell}^{\ell} \sum_{p=0}^{\infty} f_{\ell m p} Z_{\ell m p}(\boldsymbol{r}),
$$

\footnotetext{
${ }^{1}|\cdot|$ denotes the absolute value
}

where $f_{\ell m p} \triangleq\left\langle f, Z_{\ell m p}\right\rangle$ denotes the Fourier-Laguerre coefficient and forms the harmonic domain representation of the signal. A signal $f$ is said to be band-limited in the spectral region $A_{P L} \triangleq\{0 \leq \ell \leq L-1,|m| \leq \ell, 0 \leq p \leq P-1\}$ if $f_{\ell m p}=0, \forall \ell>L$ and $\forall p>P$. These functions form a $P L^{2}$ dimensional space, $\mathcal{H}_{P L}$. In the sequel, we adopt the short-hand notation $\sum_{\ell m p}^{(L, P)}=\sum_{\ell=0}^{L-1} \sum_{m=-\ell}^{\ell} \sum_{p=0}^{P-1}$ for succinct representation.

\section{Slepian Concentration Problem on the Ball}

Next, we summarize the Slepian concentration problem (on the ball) for finding the band-limited functions that have maximum energy concentration in the spatial region or the spacelimited functions that have maximum energy concentration in the spectral region [3], [9], [13].

In [9], the problem pertaining to maximization of concentration of unit energy band-limited function $f \in \mathcal{H}_{P L}$ in some spatial region $R \subset \mathbb{B}^{3}$ has been presented using a Fredholm integral equation as

$$
\left(\mathcal{S}_{R} \mathcal{S}_{P L} \mathcal{S}_{R} f\right)(\boldsymbol{r})=\lambda f(\boldsymbol{r}), \quad \boldsymbol{r} \in \mathbb{B}^{3} .
$$

Here $\mathcal{S}_{R}$ is a spatial selection operator with the kernel given by

$$
S_{R}\left(\boldsymbol{r}, \boldsymbol{r}^{\prime}\right) \triangleq I_{R}(\boldsymbol{r}) \delta\left(\boldsymbol{r}, \boldsymbol{r}^{\prime}\right),
$$

where $I_{R}(\boldsymbol{r})$ and $\delta\left(\boldsymbol{r}, \boldsymbol{r}^{\prime}\right)$ represent the indicator function for the region $R$ and Dirac delta function on the unit ball respectively [10]. We further note that $I_{R}(\boldsymbol{r})=0$ for $\boldsymbol{r} \in \mathbb{B}^{3} \backslash R$ and $I_{R}(\boldsymbol{r})=1$ for $\boldsymbol{r} \in R \subset \mathbb{B}^{3}$. $\mathcal{S}_{P L}$ in (7) is a spectral selection operator $\mathcal{S}_{P L}$ with kernel given by

$$
S_{P L}\left(\boldsymbol{r}, \boldsymbol{r}^{\prime}\right) \triangleq \sum_{\ell m p}^{(L, P)} Z_{\ell m p}(\boldsymbol{r}) \overline{Z_{\ell m p}\left(\boldsymbol{r}^{\prime}\right)}
$$

We note that the operators $\mathcal{S}_{R}$ and $\mathcal{S}_{P L}$ limit the signal to the spatial region $R$ and spectral region $A_{P L}$ respectively.

Using signal expansion in Fourier-Laguerre basis functions, given in (6), and the kernel representations in (8) and (9), the concentration problem in (7) can be equivalently formulated as an algebraic eigen-problem of size $P L^{2}$, given by

$$
\sum_{\ell^{\prime} m^{\prime} p^{\prime}}^{(L, P)} f_{\ell^{\prime} m^{\prime} p^{\prime}} \int_{R} Z_{\ell^{\prime} m^{\prime} p^{\prime}}(\boldsymbol{r}) \overline{Z_{\ell m p}(\boldsymbol{r})} d v(\boldsymbol{r})=\lambda f_{\ell m p},
$$

where $\ell, m, p \in A_{P L}$. Solving this eigenvalue problem we get $P L^{2}$ band-limited eigenfunctions. Let $f_{u}$ denote the eigenfunctions for $u \in\left[1, P L^{2}\right]$. The eigenfunctions are indexed such that $1>\lambda_{1} \geq \lambda_{2} \geq \ldots \geq \lambda_{P L^{2}}>0$ where $\lambda_{u}$ denotes the eigenvalues.

Likewise the maximization of energy concentration of a unit-energy space-limited function $h \in \mathcal{H}_{R}$, within the spectral region $A_{P L}$, is an eigenvalue problem analogous to (7) and is given by [9] as

$$
\left(\mathcal{S}_{P L} \mathcal{S}_{R} \mathcal{S}_{P L} h\right)(\boldsymbol{r})=\lambda h(\boldsymbol{r}), \quad \boldsymbol{r} \in R .
$$


Here $\mathcal{H}_{R}$ represents the space of space-limited functions with finite-energy. Since the integral equations given in (7) and (10) share the same solution $r \in R$ [9], the spacelimited eigenfunctions of (10) are similar to the band-limited eigenfunctions of (7) in the region $R$. For every band-limited eigenfunction $f_{u} \in \mathcal{H}_{P L}$, we have an associated spatiallylimited eigenfunction $h_{u}=\mathcal{S}_{R} f_{u} \in \mathcal{H}_{R}$, for $u \in\left[1, P L^{2}\right]$. The eigenvalue $0<\lambda_{u}<1$ associated with each function quantifies the concentration of energy of the space-limited eigenfunction $h_{u}$ in the spectral region $A_{P L}$ and the bandlimited eigenfunction $f_{u}$ in the region $R$, that is,

$$
\begin{array}{r}
\left\|f_{u}\right\|_{R}^{2} \equiv\left\langle f_{u}, f_{u}\right\rangle_{R} \triangleq\left\langle\mathcal{S}_{R} f_{u}, \mathcal{S}_{R} f_{u}\right\rangle=\lambda_{u}, \\
\left\|h_{u}\right\|_{P L}^{2} \triangleq\left\langle\mathcal{S}_{P L} h_{u}, \mathcal{S}_{P L} h_{u}\right\rangle=\lambda_{u} .
\end{array}
$$

\section{Problem Formulation}

The set of band-limited eigenfunctions $f_{u} \in \mathcal{H}_{P L}, u \in$ $\left[1, P L^{2}\right]$ forms orthonormal bases, whereas that of spacelimited eigenfunctions $h_{u} \in \mathcal{H}_{R}, u \in\left[1, P L^{2}\right]$ forms orthogonal bases. Let the subspace spanned by these eigenfunctions be $\mathcal{H}_{P L}$ and $\widetilde{\mathcal{H}}_{R} \subset \mathcal{H}_{R} \subset L^{2}\left(\mathbb{B}^{3}\right)$, respectively (both are finite dimensional.) Therefore, using eigenfunctions we can represent signals more economically, i.e. using less number of basis functions, than using Fourier-Laguerre basis functions, especially if the signal is band-limited and spatially concentrated in some region $R$, or if it is space-limited and spectrally concentrated in region $A_{P L}$. Practically, signals may not be entirely limited in spectral domain nor in spatial domain, and representing such signals demands bases (or eigenfunctions) that maximize the concentration of energy in both harmonic and spatial domains. In this paper, our objective is to develop basis functions which maximize the product of concentration of energy in both spectral and spatial domains.

\section{Bases with Maximal Multiplicative CONCENTRATION}

We aim to develop basis functions for a joint subspace $\mathcal{H}_{P L}+\widetilde{\mathcal{H}}_{R}$, that is, the vector sum of $\mathcal{H}_{P L}$ and $\widetilde{\mathcal{H}}_{R}$. The design of the basis functions is based upon the maximization of the product of energy concentration in spectral region $A_{P L}$ and spatial region $R$.

\section{A. Design of Optimal Basis Functions}

Since the band-limited eigenfunctions $f_{u}, u \in\left[1, P L^{2}\right]$ and the space-limited eigenfunctions $h_{u}=\mathcal{S}_{R} f_{u}, u \in\left[1, P L^{2}\right]$ are designed to have maximal concentration in spatial region $R$ and spectral region $A_{P L}$ respectively, we hereby form a new set of functions as a weighted linear combination of these eigenfunctions, as follows:

$$
g_{u}(\boldsymbol{r}) \triangleq \alpha_{u} f_{u}(\boldsymbol{r})+\beta_{u} h_{u}(\boldsymbol{r}), \quad h_{u}(\boldsymbol{r})=\left(\mathcal{S}_{R} f_{u}\right)(\boldsymbol{r}),
$$

for $u \in\left[1, P L^{2}\right]$, where the weights $\alpha_{u}$ and $\beta_{u}$ are optimally chosen to maximize the product of concentration of energy of the function $g_{u}$ in the spectral region $A_{P L}$ as well as in the spatial region $R$. Since $f_{u} \in \mathcal{H}_{P L}$ and $h_{u} \in \widetilde{\mathcal{H}}_{R}$, we note that every $g_{u} \in \mathcal{H}_{P L}+\mathcal{H}_{R}$. The function $g_{u}$ is a unit energy function. The space-limited and band-limited eigenfunctions satisfy the following orthogonality relations [15]

$$
\begin{aligned}
& \left\|h_{u}\right\|_{R}^{2}=\left\langle h_{u}, h_{v}\right\rangle_{R}=\left\langle f_{u}, h_{v}\right\rangle_{R}=\left\|f_{u}\right\|_{R}^{2}=\delta_{u, v} \lambda_{u}, \\
& \left\|h_{u}\right\|_{P L}^{2}=\lambda_{u}^{2}, \quad\left\|f_{u}\right\|_{P L}^{2}=\left\langle f_{u}, f_{u}\right\rangle=\delta_{u, v}
\end{aligned}
$$

for $u, v \in\left[1, P L^{2}\right]$, where $\delta_{u, v}$ is the Kronecker delta. Next determine the weights $\alpha_{u}$ and $\beta_{u}$.

Theorem 1. For any unit energy function $g_{u} \in \mathcal{H}_{P L}+\widetilde{\mathcal{H}}_{R}$, the product of concentration of energy in the spectral region $A_{P L}$ and that in the spatial region $R \subset \mathbb{B}^{3}$ becomes maximum when $\alpha_{u}$ and $\beta_{u}$ have the values:

$$
\alpha_{u, 1}=X, \quad \beta_{u, 1}=\frac{X}{\sqrt{\lambda_{u}}}, \quad \alpha_{u, 2}=Y, \quad \beta_{u, 2}=-\frac{Y}{\sqrt{\lambda_{u}}},
$$

where $X=\left(2+2 \sqrt{\lambda_{u}}\right)^{-1 / 2}$ and $Y=\left(2-2 \sqrt{\lambda_{u}}\right)^{-1 / 2}$.

Proof. This problem can be formulated as a constrained maximization problem given by

$$
\text { maximize }\left\|g_{u}\right\|_{R}^{2}\left\|g_{u}\right\|_{P L}^{2} \text { subject to }\left\|g_{u}\right\|^{2}=1 \text {, }
$$

with the Lagrangian $\mathcal{L}$ given as

$$
\mathcal{L}=\left\|g_{u}\right\|_{R}^{2}\left\|g_{u}\right\|_{P L}^{2}+\gamma\left(\left\|g_{u}\right\|^{2}-1\right)
$$

Noting $\left\|g_{u}\right\|_{R}^{2}=\lambda_{u}\left(\alpha_{u}+\beta_{u}\right)^{2}$ and $\left\|g_{u}\right\|_{P L}^{2}=\left(\alpha_{u}+\beta_{u} \lambda_{u}\right)^{2}$ yields the two solutions for $\alpha_{u}$ and $\beta_{u}$ given in (15).

For the two pairs of weights $\alpha_{u}$ and $\beta_{u}$ obtained in Theorem 1, we define a set of functions as

$$
g_{u, j}(\boldsymbol{r})=\alpha_{u, j} f_{u}(\boldsymbol{r})+\beta_{u, j}\left(\mathcal{S}_{R} f_{u}\right)(\boldsymbol{r}),
$$

for $u \in\left[1, P L^{2}\right], j=[1,2]$ with the concentration of energy given by,

$$
\left\|g_{u, j}\right\|_{R}^{2}=\left\|g_{u, j}\right\|_{P L}^{2}=\frac{1-(-1)^{j} \sqrt{\lambda_{u}}}{2}, \quad j=1,2,
$$

which we obtain by substituting the values for $\alpha_{u, j}$ and $\beta_{u, j}$ in their respective norm functions.

We yet need to check the completeness of the basis functions in (16) for the joint subspace $\mathcal{H}_{P L}+\widetilde{\mathcal{H}}_{R}$. From (14) it can be seen that the functions $g_{u, 1}$ and $g_{v, 2}$ for $u, v \in\left[1, P L^{2}\right], u \neq v$ are orthonormal (unit energy constraint on $g_{u, j}$ ) for any values of $\alpha_{u, 1}, \alpha_{v, 2}, \beta_{u, 1}$ and $\beta_{v, 2}$. Also from (15), it can be easily shown that $g_{u, 1}$ and $g_{u, 2}$ become orthonormal for each $u \in\left[1, P L^{2}\right]$. The joint subspace has dimension $2 P L^{2}$ (each of the subspaces $\widetilde{\mathcal{H}}_{R}$ and $\mathcal{H}_{P L}$ is of dimension $P L^{2}$ ), therefore, the $2 P L^{2}$ number of orthonormal functions $g_{u, j} \in \mathcal{H}_{P L}+\widetilde{\mathcal{H}}_{R}$ for $u \in\left[1, P L^{2}\right]$, and $j=1,2$ completely span the joint subspace $\mathcal{H}_{P L}+\widetilde{\mathcal{H}}_{R}$. 


\section{B. Integral Operator Formulation}

We now have a set of functions which form the bases for the subspace $\mathcal{H}_{P L}+\widetilde{\mathcal{H}}_{R}$. These functions are hereby referred as the optimal basis functions for the ball. They are optimally designed based upon the maximization of the product of measures of energy concentration in the spectral region $A_{P L}$ and the spatial region $R$. From the set of optimal basis functions, the functions of more significance are the ones that are greatly concentrated in the spectral or spatial region of interest. From (17), we can see that energy concentration of each $g_{u, j}$ is same in both the spectral and spatial regions. However, the functions do not have simultaneous maximal energy concentration in both the spectral and spatial domains. Using the Fredholm integral equation, we develop a linear integral operator which will serve as a measure for the simultaneous concentration of energy in the spatial and spectral regions of interest.

Theorem 2. The function $y \in L^{2}\left(\mathbb{B}^{3}\right)$, that maximizes the product of concentration of energy in some spectral region $A_{P L}$ and that in the spatial region $R \subset \mathbb{B}^{3}$, is an eigenfunction of a linear integral operator $\mathcal{S}_{M}$ with kernel $S_{M}\left(\boldsymbol{r}, \boldsymbol{r}^{\prime}\right)$ given by

$$
S_{M}\left(\boldsymbol{r}, \boldsymbol{r}^{\prime}\right)=\frac{I_{R}(\boldsymbol{r})+I_{R}\left(\boldsymbol{r}^{\prime}\right)}{2} \sum_{\ell m p}^{(L, P)} Z_{\ell m p}(\boldsymbol{r}) \overline{Z_{\ell m p}\left(\boldsymbol{r}^{\prime}\right)},
$$

and the eigenvalue, denoted by $\mu$, of the eigenfunction $y$ such that $\left(\mathcal{S}_{M} y\right)(\boldsymbol{r})=\mu y(\boldsymbol{r})$ is given by

$$
\mu=\|y\|_{R}^{2}\left(2\|y\|_{R}^{2}-1\right)
$$

Proof. We consider $U(\boldsymbol{r})=y(\boldsymbol{r})+\epsilon z(\boldsymbol{r})$, where $y$ is a unit energy function, $z \in L^{2}\left(\mathbb{B}^{3}\right)$ and $\epsilon \in \mathbb{R}$ quantifies the small disturbance in the solution. The concentration of energy in the spectral region $A_{P L}$ and the spatial region $R$ is given as $\|y\|_{P L}^{2}$ and $\|y\|_{R}^{2}$ respectively. By applying the variational principle, we find the function $y$ for which the product of concentration of energy in the the two regions (i.e., spatial and spectral), defined as

$$
V=\frac{\|U\|_{R}^{2}}{\|U\|^{2}} \frac{\|U\|_{P L}^{2}}{\|U\|^{2}}
$$

is maximized. Here the factor $\|U\|^{2}$ is used to ensure unit energy is normalized. As $0 \leq V \leq 1$, we maximize $V$ by maximizing $\log V$ as

$$
\left.\frac{d}{d \epsilon}(\log V)\right|_{\epsilon=0}=\left.\frac{d}{d \epsilon}\left(\log \frac{\|U\|_{R}^{2}}{\|U\|^{2}}+\log \frac{\|U\|_{P L}^{2}}{\|U\|^{2}}\right)\right|_{\epsilon=0}=0 .
$$

By substituting $\|U\|_{R}=\left\langle\mathcal{S}_{R} U, \mathcal{S}_{R} U\right\rangle$ and $\|U\|_{P L}=$ $\left\langle\mathcal{S}_{P L} U, \mathcal{S}_{P L} U\right\rangle$, we can simplify the above equation as

$\frac{1}{2\|y\|_{R}^{2}}\left(\mathcal{S}_{R}(y z)\right)(\boldsymbol{r})+\frac{1}{2\|y\|_{L}^{2}}\left(\mathcal{S}_{P L}(y z)\right)(\boldsymbol{r})=y(\boldsymbol{r}) z(\boldsymbol{r}) \Psi$

which holds for every $z \in L^{2}\left(\mathbb{B}^{3}\right)$.
Using the method described in [15] and employing the orthonormality of Fourier-Laguerre basis functions, it can be shown that

$$
\begin{aligned}
\left\langle\mathcal{S}_{R} \mathcal{S}_{P L} y, \mathcal{S}_{R} y\right\rangle & =\overline{\left\langle\mathcal{S}_{P L} \mathcal{S}_{R} y, \mathcal{S}_{P L} y\right\rangle} \\
& =\sum_{\ell m p}^{(L, P)} y_{\ell m p}\left\langle Z_{\ell m p}, y\right\rangle_{R},
\end{aligned}
$$

and we conclude that

$$
\|y\|_{R}^{2}=\|y\|_{L}^{2}
$$

After rearranging terms we arrive at the result

$$
\begin{aligned}
& 2\|y\|_{R}^{2}\left(2\|y\|_{R}^{2}-1\right) y(\boldsymbol{r})=\left(\mathcal{S}_{P L} \mathcal{S}_{R} y+\mathcal{S}_{P L} y\right)(\boldsymbol{r}), \boldsymbol{r} \in R, \\
& 2\|y\|_{R}^{2}\left(2\|y\|_{R}^{2}-1\right) y(\boldsymbol{r})=\left(\mathcal{S}_{P L} \mathcal{S}_{R} y\right)(\boldsymbol{r}), \boldsymbol{r} \in \mathbb{B}^{3} \backslash R .
\end{aligned}
$$

Using the kernels for the operators $\mathcal{S}_{R}$ and $\mathcal{S}_{P L}$, given in (8) and (9), respectively, we conclude that $\mathcal{S}_{M} y(\boldsymbol{x})=\mu y(\boldsymbol{x})$, where (18) and (19) represent the kernel of the $\mathcal{S}_{M}$ operator and its eigenvalue respectively.

Now we show that the integral operator $\mathcal{S}_{M}$ has eigenfunctions given by the basis functions $g_{u, j}, u \in\left[1, P L^{2}\right], j=1,2$.

Theorem 3. Optimal basis functions $g_{u, j}$, given in (16), are the only eigenfunctions of the operator $\mathcal{S}_{M}$.

Proof. First we show how the optimal basis functions form eigenfunctions of the operator $\mathcal{S}_{M}$. By substituting function $y$ in (22) with any basis function, we obtain $\left(\mathcal{S}_{M} g_{u, j}\right)(\boldsymbol{r})=$ $\mu_{u, j} g_{u, j}(\boldsymbol{r})$. Here the eigenvalue $\mu_{u, j}=\left\|g_{u, j}\right\|_{R}^{2}\left(2\left\|g_{u, j}\right\|_{R}^{2}-\right.$ 1 ) is a measure of concentration of energy in the spectral as well as spatial regions. To prove that the optimal basis functions are the only eigenfunctions of the operator, we first note that the operator $\mathcal{S}_{M}$ is self-adjoint which directly follows from the definition of its kernel $S_{M}\left(\boldsymbol{r}, \boldsymbol{r}^{\prime}\right)$ given in (18). It can also be shown that $\mathcal{S}_{M}$ is a Hilbert-Schmidt operator. The self-adjointness and square-integrability together with the fact that zero is not an eigenvalue of the operator $\mathcal{S}_{M}$ imply that eigenfunctions of the operator form complete basis functions for $\mathcal{H}_{P L}+\widetilde{\mathcal{H}}_{R}$. This, together with the prior discussion that optimal basis functions are a complete set of basis functions for the subspace $\mathcal{H}_{P L}+\widetilde{\mathcal{H}}_{R}$, completes the proof of this theorem.

There are several consequences of Theorem 3. This implies that $\mathcal{S}_{M}$ is a projection operator which projects a signal $x \in$ $L^{2}\left(\mathbb{B}^{3}\right)$ to the subspace $\mathcal{H}_{P L}+\widetilde{\mathcal{H}}_{R}$. Furthermore, it maps any signal in $\mathcal{H}_{P L}+\widetilde{\mathcal{H}}_{R}$ to a signal in $\mathcal{H}_{P L}+\widetilde{\mathcal{H}}_{R}$. We review the properties of optimal basis functions in the next section.

\section{Properties}

Orthogonality of Eigenfunctions: The optimal basis functions $g_{u, j}$ are orthogonal over both $\mathcal{H}_{P L}$ and $\mathcal{H}_{R}$, i.e.,

$\left\langle g_{u, j}, g_{v, j}\right\rangle_{R}=\left\|g_{u, j}\right\|_{R}^{2} \delta_{u, v}, \quad\left\langle g_{u, j}, g_{v, j}\right\rangle_{P L}=\left\|g_{u, j}\right\|_{P L}^{2} \delta_{u, v}$.

Spectrum of Eigenvalues: For eigenvalues indexed as $1>$ $\lambda_{u} \geq \lambda_{v}>0$, we have the following distribution for the 
product of concentration of energy in the spectral and spatial domains for optimal basis functions for $u<v$ and $u, v \in$ $\left[1, P L^{2}\right]$ :

$$
\begin{aligned}
1 & >\left\|g_{u, 1}\right\|_{R}^{2}\left\|g_{u, 1}\right\|_{P L}^{2}>\left\|g_{v, 1}\right\|_{R}^{2}\left\|g_{v, 1}\right\|_{P L}^{2}>0.25, \\
0.25 & >\left\|g_{u, 2}\right\|_{R}^{2}\left\|g_{u, 2}\right\|_{P L}^{2}>\left\|g_{v, 2}\right\|_{R}^{2}\left\|g_{v, 2}\right\|_{P L}^{2}>0 .
\end{aligned}
$$

For $j=1$, the eigenvalue $\mu_{u, j}$ decreases monotonically to 0 as $u$ increases from 1 to $P L^{2}$, whereas for $j=2$, it decreases from some negative value to a minima and later increases to 0 as $u$ goes from 1 to $P L^{2}$.

\section{Signal Representation in Optimal Basis Function}

We arrange the optimal basis functions $g_{u, j}, u \in\left[1, P L^{2}\right]$, $j=1,2$, as $\psi_{1}, \psi_{2}, \ldots, \psi_{2 P L^{2}}$, in decreasing order of eigenvalues' magnitudes. As mentioned before, we can project any signal $x \in L^{2}\left(\mathbb{B}^{3}\right)$ to the subspace $\mathcal{H}_{P L}+\widetilde{\mathcal{H}}_{R}$ using the optimal basis functions as

$$
x(\boldsymbol{r})=\sum_{u=1}^{2 P L^{2}}\left\langle x, \psi_{u}\right\rangle \psi_{u}(\boldsymbol{r}),
$$

where the inner product is evaluated as

$$
\begin{aligned}
& \left\langle x, \psi_{u}\right\rangle=\sum_{\ell m p}^{(L, P)} x_{\ell m p}\left(\psi_{u}\right)_{\ell m p} \\
& =\sum_{\ell m p}^{(L, P)} x_{\ell m p}\left(\alpha_{u}\left(f_{u}\right)_{\ell m p}+\beta_{u} \sum_{\ell^{\prime} m^{\prime} p^{\prime}}^{(L, P)}\left(f_{u}\right)_{\ell^{\prime} m^{\prime} p^{\prime}} K_{\ell m p, \ell^{\prime} m^{\prime} p^{\prime}}\right)
\end{aligned}
$$

and $K_{\ell m p, \ell^{\prime} m^{\prime} p^{\prime}}=\int_{R} Z_{\ell^{\prime} m^{\prime} p^{\prime}}(\boldsymbol{r}) \overline{Z_{\ell m p}(\boldsymbol{r})} d v(\boldsymbol{r})$ [10]. If the signal $x$ is spatially concentrated in the region $R$ and spectrally concentrated in the region $A_{P L}$, it is expected that we can represent it using lesser number of terms since the inner product $\left\langle x, \psi_{u}\right\rangle$ decreases to 0 as $u$ goes from 1 to $2 P L^{2}$.

\section{CONCLUSIONS}

In this paper, we have presented the design of complete orthonormal optimal basis functions for the unit ball with simultaneous maximal energy concentration in both the spectral (harmonic) and spatial domain. We design optimal basis as a linear combination of spectrally concentrated space-limited functions and spatially concentrated band-limited functions. The proposed developments have been presented in the form of 3 theorems. First, we have optimally designed the weights to maximize the product of concentration of energy in harmonic and spatial domains. We have also shown that the proposed optimal basis functions span the subspace given by the vector sum of subspace of space-limited spectrally concentrated functions and subspace of band-limited spatially concentrated functions. We have also formulated the integral operator as a projection operator that projects the signal on the ball to the vector sum of subspaces and maximizes the product of energy concentrations in harmonic as well as spatial domains. We have also shown that the proposed optimal basis are the only eigenfunctions of the proposed integral operator. We reviewed some of the properties of proposed optimal bases. Further analysis of the proposed basis and application to the problems of signal estimation and localized spectral analysis are the subjects of future work.

\section{ACKNOWLEDGMENT}

Rodney A. Kennedy is supported by the Australian Research Councils Discovery Projects funding scheme (Project no. DP150101011).

\section{REFERENCES}

[1] B. Leistedt and J. D. McEwen, "Exact wavelets on the ball," IEEE Trans. Sig. Proc., vol. 60, no. 12, pp. 6257-6269, 2012.

[2] F. J. Simons and D. V. Wang, "Spatiospectral concentration in the cartesian plane," Intern. J. Geomath., vol. 2, no. 1, pp. 1-36, 2011.

[3] A. Albertella, F. Sansò, and N. Sneeuw, "Band-limited functions on a bounded spherical domain: the Slepian problem on the sphere," $J$. Geodesy, vol. 73, no. 9, pp. 436-447, Jun. 1999.

[4] Y. F. Alem, Z. Khalid, and R. A. Kennedy, "3D spatial fading correlation for uniform angle of arrival distribution,” IEEE Commun. Lett., vol. 19, pp. 1073-1076, June 2015.

[5] A. P. Bates, Z. Khalid, and R. A. Kennedy, "An optimal dimensionality sampling scheme on the sphere with accurate and efficient spherical harmonic transform for diffusion MRI," IEEE Signal Process. Lett., vol. 23, pp. 15-19, January 2016.

[6] F. J. Simons, I. Loris, G. Nolet, I. C. Daubechies, S. Voronin, P. A. Vetter J. Charlty, and C. Vonesch, "Solving or resolving global tomographic models with spherical wavelets, and the scale and sparsity of seismic heterogeneity," Geophys. J. Int., vol. 187, pp. 969-988, 2011.

[7] F. Lanusse, A. Rassat, , and J.-L. Starck, "Spherical 3D isotropic wavelets," Astron. \& Astrophys., vol. 540, p. A92, Apr. 2012.

[8] D. Slepian and H. O. Pollak, "Prolate spheroidal wave functions, Fourier analysis and uncertainity-I," Bell Systems Technical Journal, vol. 40, pp. 43-63, Jan. 1961.

[9] F. J. Simons, F. A. Dahlen, and M. A. Wieczorek, "Spatiospectral concentration on a sphere," SIAM Rev., vol. 48, no. 3, pp. 504-536, 2006.

[10] Z. Khalid, R. A. Kennedy, and J. D. McEwen, "Slepian spatial-spectral concentration on the ball," arXiv preprint arXiv:1403.5553, 2014.

[11] Z. Khalid, P. Sadeghi, R. A. Kennedy, and S. Durrani, "Spatially varying spectral filtering of signals on the unit sphere," IEEE Trans. Signal Process., vol. 61, no. 3, pp. 530-544, Feb. 2013.

[12] M. A. Wieczorek and F. J. Simons, "Minimum variance multitaper spectral estimation on the sphere," J. Fourier Anal. Appl., vol. 13, no. 6, pp. 665-692, 2007.

[13] 1 , "Localized spectral analysis on the sphere," Geophys. J. Int., vol. 162, no. 3, pp. 655-675, Sep. 2005.

[14] Q. Jin, Z. Luo, and K. M. Wong, "An optimum complete orthonormal basis for signal analysis and design," IEEE Trans. Inf. Theory, vol. 40, no. 3, pp. 732-742, May 1994.

[15] Z. Khalid and R. A. Kennedy, "Maximal multiplicative spatial-spectral concentration on the sphere: Optimal basis," in 2015 IEEE International Conference on Acoustics, Speech and Signal Processing (ICASSP). IEEE, 2015, pp. 4160-4164. 